\title{
The 21st-century challenge to neurocritical care: the rise of the superbug Acinetobacter baumannii. A meta-analysis of the role of intrathecal or intraventricular antimicrobial therapy in reduction of mortality
}

\author{
Nasser Mohammed, MD, MCh, Amey R. Savardekar, MCh, Devi Prasad Patra, MD, MCh, \\ Vinayak Narayan, MD, MCh, and Anil Nanda, MD, MPH \\ Department of Neurosurgery, Louisiana State University Health Sciences Center, Shreveport, Louisiana
}

OBJECTIVE Neurosurgical infections due to multidrug-resistant organisms have become a nightmare that neurosurgeons are facing in the 21 st century. This is the dawn of the so-called postantibiotic era. There is an urgent need to review and evaluate ways to reduce the high mortality rates due to these infections. The present study evaluates the efficacy of combined intravenous plus intrathecal or intraventricular (IV + IT) therapy versus only intravenous (IV) therapy in treating postneurosurgical Acinetobacter baumannii infections.

METHODS The authors performed a meta-analysis of all peer-reviewed studies from the PubMed, Cochrane Library database, ScienceDirect, and EMBASE in accordance with the PRISMA (Preferred Reporting Items for Systematic Reviews and Meta-Analyses) guidelines. Five studies were finally included in the present analysis: 126 patients were studied who had postneurosurgical $A$. baumannii infection. The Cochrane collaboration tool was used to evaluate risk of bias, and a test of heterogeneity was performed. The $\mathrm{I}^{2}$ statistic was calculated. The patients were divided into 2 groups: the IV group received only intravenous therapy and the IV + IT group received both intravenous and intrathecal or intraventricular antimicrobial therapy. The outcome was mortality attributed specifically to $A$. baumannii infection in postneurosurgical cases. The pooled data were analyzed using the Cochran-Mantel-Haenszel method in a fixed-effects model.

RESULTS The total number of patients in the IV-only group was 73 , and the number of patients in the IV + IT group was 53. The mean duration of intravenous therapy was 27 days. The mean duration of intrathecal colistin was 21 days. The intravenous dose of colistin ranged from 3.75 to $8.8 \mathrm{MIU}$ per day. The dose of intrathecal colistin ranged between 125,000 and 250,000 IU per day. The overall calculated odds ratio for mortality for the IV + IT group after pooling the data was $0.16(95 \% \mathrm{Cl} 0.06-0.40, \mathrm{p}<0.0001)$. The patients who received IV + IT therapy had an $84 \%$ lower risk of dying due to the infection compared with those who received only IV therapy.

CONCLUSIONS There is an $84 \%$ lower risk of mortality in patients who have been treated with combined intrathecal or intraventricular plus intravenous antimicrobial therapy versus those who have been treated with intravenous therapy alone. The intrathecal or intraventricular route should be strongly considered when dealing with postneurosurgical multidrug-resistant $A$. baumannii infections.

https://thejns.org/doi/abs/10.3171/2017.8.FOCUS17443

KEY WORDS Acinetobacter baumannii; multidrug resistance; intraventricular; intrathecal; postoperative neurosurgical infection; colistin

$\mathrm{P}$ OSTNEUROSURGICAL infections due to multidrug-resistant (MDR) organisms have become a nightmare for 21st-century neurosurgeons. This period has already been called the dawn of the postantibiotic era..$^{21}$ The accidental discovery of penicillin by Sir Alexander Fleming heralded the era of antibiotics and of human dominance over pathogens. Approximately 1 century later, at least in critical care settings, the pathogens seem to be dominating again. Acinetobacter baumannii is one such pathogen that has become the scourge of neurosurgical ICUs worldwide,

ABBREVIATIONS CMS = colistimethate sodium; IV + IT = intravenous plus intrathecal or intraventricular; MDR = multidrug-resistant; PRISMA = Preferred Reporting Items for Systematic Reviews and Meta-Analyses.

SUBMITTED July 3, 2017. ACCEPTED August 7, 2017.

INCLUDE WHEN CITING DOI: 10.3171/2017.8.FOCUS17443. 
resulting in several global outbreaks. ${ }^{27}$ The mortality rate due to MDR A. baumannii meningitis can range from $15 \%$ to $72.7 \% .^{17,21,26}$

The data from SENTRY (Antimicrobial Surveillance Program), MYSTIC (Meropenem Yearly Susceptibility Test Information Collection), and other sources have reported the emergence of MDR A. baumannii from Europe, North America, Argentina, Brazil, China, Taiwan, Hong Kong, Japan, Korea, and even remote islands like Tahiti. ${ }^{21}$ It has been nicknamed "Iraqibacter" due to the increased number of nosocomial infections in military hospitals during Operation Iraqi Freedom. ${ }^{1}$ In this evolutionary arms race, there is an urgent need to review, evaluate, and find new ways to tackle this difficult problem. The intrathecal or intraventricular (IT) administration of antibiotics has been tried as a last resort in many cases of severe neuro-infections not responding to traditional intravenous (IV) regimens. ${ }^{10}$ This analysis is an attempt to review the role of intrathecal or intraventricular antibiotic therapy and its effect on the mortality rate.

\section{Methods}

The aim of the study was to review the benefit of combined IV + IT therapy over exclusive IV therapy. The outcome studied was mortality attributed specifically to $A$. baumannii meningitis. A detailed protocol about the literature search, inclusion and exclusion criteria, selection of cases, and statistical methodology was developed. The guidelines laid out by the Preferred Reporting Items for Systematic Reviews and Meta-Analyses (PRISMA) were followed. Two investigators (N.M. and V.N.) performed the literature search. To minimize selection bias, 2 reviewers (A.S. and D.P.) reviewed the articles independently. In case of doubt, a mutual consensus was reached after discussion.

\section{Literature Search Strategy}

A computerized search of titles and abstracts from January 1960 to May 2017 was performed in PubMed, Cochrane Library database, ScienceDirect, and EMBASE. A combination of key word searches was made to build a search strategy. The non-English articles were also searched. Two non-English articles were reviewed after translation. An independent Web search was made with the key words to include any unpublished literature. The reference lists of final selected articles were examined and reviewed (Fig. 1). Key words that were used to build the search directory were "Acinetobacter baumannii," "Neurosurgery," "postoperative," and "infections." The example of a PubMed search directory is as follows: ("acinetobacter baumannii”"[MeSH Terms]) OR ("acinetobacter"[All Fields] AND "baumannii"[All Fields]) OR “acinetobacter baumannii"[All Fields]) AND ("neurosurgical procedures"[MeSH Terms] OR ("neurosurgical"[All Fields] AND "procedures"[All Fields]) OR "neurosurgical procedures"[All Fields] OR "neurosurgery"[All Fields] OR "neurosurgery" [MeSH Terms]). Additional search terms used were "intrathecal," "intraventricular," "colistin," "polymyxin," "meningitis," "craniotomy," and "multidrug resistant."

\section{Risk of Bias Assessment}

Two investigators independently reviewed all the articles to eliminate selection bias. Disagreements were sorted out after discussing the merit of each study. The heterogeneity of the selected studies was assessed with the $\mathrm{I}^{2}$ statistic. The $\mathrm{I}^{2}$ value of the present study was $28 \%$. A funnel plot was charted to evaluate bias.

\section{Inclusion and Exclusion Criteria}

Patients who had undergone any neurosurgical procedure of the brain and spine and who subsequently developed CNS infection with A. baumannii in the postoperative period were considered. Only the studies that had comparative cohorts of patients undergoing IV and IV + IT therapy were selected. The studies with incomplete data about treatment and outcome were excluded, and the case reports were excluded. The groups were matched for their similarities to remove confounding variables. Postoperative meningitis was defined according to the Centers for Disease Control's definition. Infection was positive if at least 1 of the following 2 criteria was fulfilled: 1) the isolation of Acinetobacter from the CSF; and 2) fever $>38^{\circ} \mathrm{C}$ in the absence of any other recognizable cause - with any of the following: increased leukocyte count $>10 / \mathrm{mm}^{3}$ with $>$ $50 \%$ polymorphs; increased protein $(>45 \mathrm{mg} / \mathrm{dl})$ in CSF; and/or decreased levels of glucose $(<40 \mathrm{mg} / \mathrm{dl})$ in CSF. A positive culture from CSF without clinical symptoms and normal results on CSF analysis was not considered an infection. Therapeutic cure was defined as resolution of signs and symptoms, with clinical improvement and no growth in 2 successive CSF cultures.

\section{Statistical Analysis}

The test for heterogeneity was performed and the $\mathrm{I}^{2}$ value evaluated. The data were analyzed with the fixedeffects model due to acceptable heterogeneity. The odds ratios with the respective confidence intervals were calculated for each study. The Cochran-Mantel-Haenszel method was used to generate an estimate of an association between the study groups. The data type was dichotomous and was expressed as an odds ratio with $95 \%$ confidence interval. A forest plot was charted and analyzed. The overall odds ratio and risk estimation was then made by summarizing all the studies. The studies were weighted according to their sample sizes. Statistical analysis was done using the Review Manager computer program (RevMan version 5.3; Copenhagen: Nordic Cochrane Centre, the Cochrane Collaboration, 2014).

\section{Results}

Five studies with 126 patients were selected (Table 1). All patients had undergone a neurosurgical procedure or procedures and had tested positive for infection with $A$. baumannii. The selected studies were matched to evaluate homogeneity. The test for homogeneity was performed, and the $I^{2}$ value was $28 \%$ (Fig. 2). The 2 groups were called "IV" for the patients who received intravenous antibiotics only, and "IV + IT" for those who received intravenous and intrathecal antibiotics. The total number of patients in the IV-only group was 73 , and the number of patients 


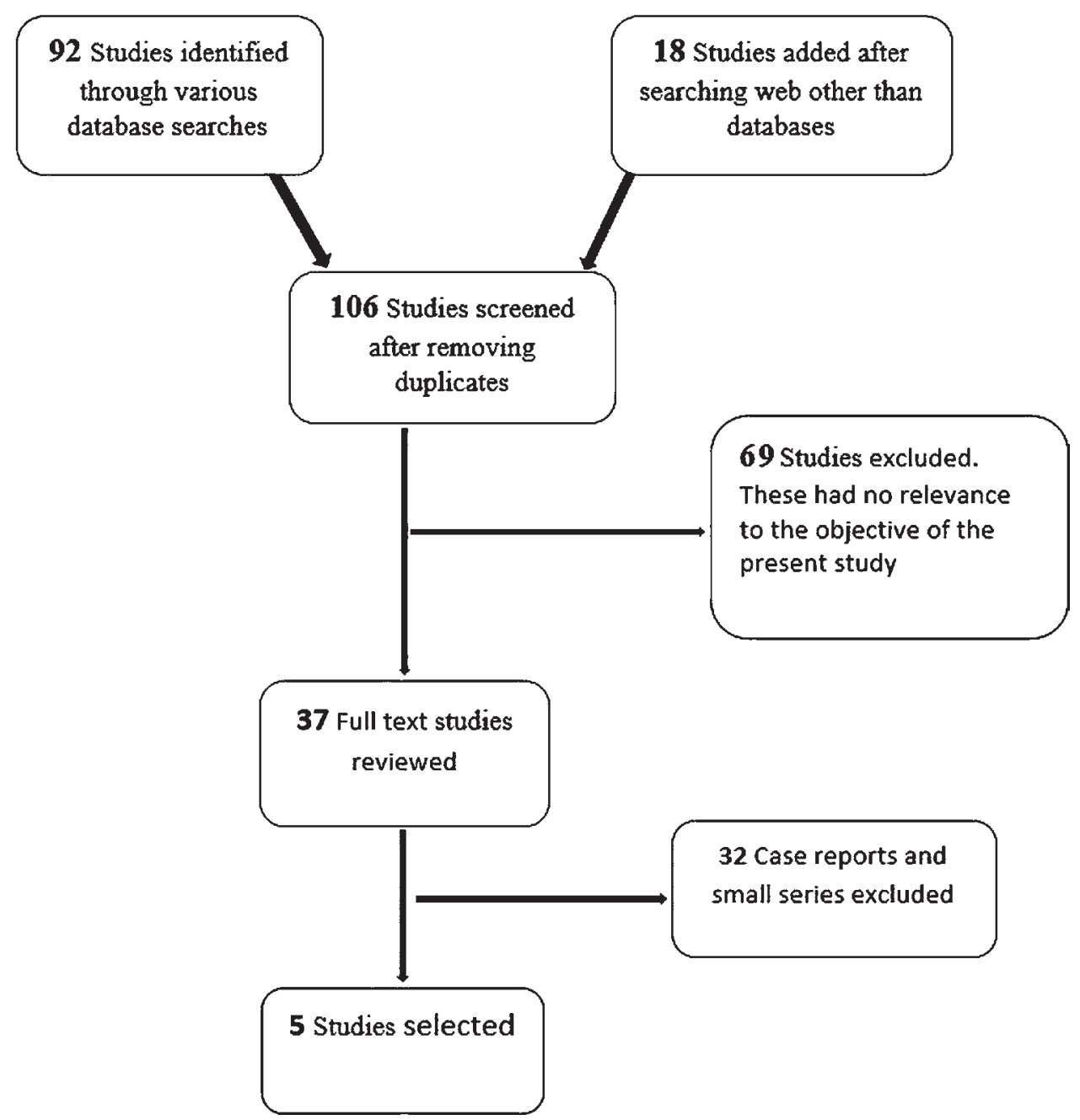

FIG. 1. The PRISMA flow diagram of systematic literature search.

in the IV + IT group was 53. The mean age in the study cohort was 48.8 years. The male/female ratio was 2.1 . The underlying disease was traumatic brain injury in 35 cases, intracerebral bleed in 56 cases, and brain neoplasm in 20 cases. The intravenous dose of colistin ranged from 3.75 to $8.8 \mathrm{MIU}$ per day, and the mean duration of IV therapy was 27 days. The dose of intrathecal colistin ranged from 125,000 to $250,000 \mathrm{IU}$ per day, and the mean duration of intrathecal colistin was 21 days (Table 2).

The data from all the subgroups in the 5 studies were analyzed by the Cochran-Mantel-Haenszel method and charted in a forest plot (Fig. 3). The overall calculated odds ratio for mortality after pooling all the data was 0.16 (95\% CI $0.06-0.40, \mathrm{p}<0.0001)$ for the IV + IT group. The patients who received IV + IT therapy had an $84 \%$ lower risk of dying due to infection compared with those who received only IV therapy. This is a very significant risk reduction for the cohorts that received IV + IT therapy.

\section{Discussion}

Acinetobacter baumannii (from the Greek word "akinetos," meaning nonmotile) was first isolated by the
Dutch microbiologist Beijerinck in 1911 from soil, as described in Antunes et al. ${ }^{2}$ From its low profile of an opportunistic organism with low virulence, ${ }^{20}$ it has shot into prominence as one of the foremost concerns of the critical care community today.

"Multidrug resistance" in literal terms means resistance to $<1$ antimicrobial agent. The scientific definition is "resistance to three or more anti-microbial classes." 15 The antimicrobial resistance of $A$. baumannii significantly increased worldwide between 2004 and 2009.12 The evolution of carbapenem-resistant A. baumannii has been the most worrying trend. Imipenem resistance in $A$. baumannii has reached $>50 \%$ from a worldwide collection between 2005 and 2009. The once-discarded antibiotics have found new relevance in this context. The most important among them in the fight with A. baumannii is colistin, also known as polymixin $\mathrm{E}$, which is an antibiotic produced by some strains of the bacterium Paenibacillus polymyxa. Structurally they are cyclic polypeptides. ${ }^{6} \mathrm{Co}-$ listin is given in the form of colistimethate sodium (CMS), which has fewer adverse reactions than colistin.

The pharmacokinetics of drug entry into the CNS is a 
TABLE 1. Details of the patients included in the meta-analysis of MDR A. baumannii

\begin{tabular}{|c|c|c|c|c|c|}
\hline Variable & Fotakopoulos et al., $2016^{*}$ & De Bonis et al., 2016 & Tuon et al., 2010 & Rodriguez et al., 2008 & Moon et al., 2013 \\
\hline Type of study & Retrospective & Retrospective & Retrospective & Retrospective & Retrospective \\
\hline No. of patients & 24 & 18 & $19 \dagger$ & 46 & 21 \\
\hline Mean age in yrs (range) & 49 & 52.05 & $43(21-91)$ & $44(15-78)$ & $56(20-83)$ \\
\hline Sex, M/F & $13 / 11$ & $10 / 8$ & $12 / 7$ & $34 / 12$ & $15 / 6$ \\
\hline \multicolumn{6}{|l|}{ Diagnosis } \\
\hline TBI & $15(62.5 \%)$ & $2(11.1 \%)$ & $4(21 \%)$ & $11(25.5 \%)$ & $3(13.6 \%)$ \\
\hline $\mathrm{SAH}$ & NA & $2(11.1 \%)$ & NA & NA & NA \\
\hline $\mathrm{ICH}$ & $16(66.6 \%)$ & $5(27.7 \%)$ & NA & $23(51 \%)$ & $12(54.5 \%)$ \\
\hline Brain tumors & $3(12.5 \%)$ & $4(22.2 \%)$ & & $10(23 \%)$ & $3(13.6 \%)$ \\
\hline No. of EVDs & NA & 6 & 12 & NA & 15 \\
\hline VPS & NA & $4(22.2 \%)$ & & NA & 4 \\
\hline $\begin{array}{l}\text { Intrathecal or intraventricular } \\
\text { dose of colistin, } I U \times 10^{3}\end{array}$ & $170 \pm 0.4$ & $125-250$ & NA & NA & 125 \\
\hline Duration of IT dose in days & $16.0 \pm 8.3$ & $26(14-40)$ & NA & NA & NA \\
\hline IV dose of colistin, IU ×106 & $8.8 \pm 0.1$ & NA & NA & NA & 3.75 \\
\hline Duration of IV dose in days & $16.0 \pm 8.3$ & NA & NA & 17 & NA \\
\hline
\end{tabular}

EVD = external ventricular drain; ICH = intracerebral hemorrhage; $N A=$ not available; $S A H$ = subarachnoid hemorrhage; $T B I=$ traumatic brain injury; VPS = ventriculoperitoneal shunt.

* Data on doses are expressed as the mean \pm SD.

$\dagger$ Two patients were not included in the statistical analysis due to reported contamination and/or colonization.

complex process. It depends on the molecular size, electric charge, lipid solubility, plasma protein binding ability, the affinity to the active transport system, CSF flow, and meningeal inflammation. ${ }^{19}$ There is a wide variation from one patient to another in the CSF concentration of the drug, despite giving the same dosage. James et al. found that the concentration of drug in CSF in patients receiving the maximum dosage of intravenous antibiot- ics alone was for the most part $\leq 5 \mu \mathrm{g} / \mathrm{ml}$ and $>5 \mu \mathrm{g} /$ $\mathrm{ml}$ for drugs given by the intraventricular route. ${ }^{8}$ The colistin penetration into CSF may be as low as 5\% $\%^{16}$ to $25 \% .{ }^{8}$ The poor penetration into CSF may be the reason for very high mortality rates in the subgroup not receiving intrathecal therapy. The risk of nephrotoxicity with polymyxin therapy ranges from $10 \%$ to $18 \% .^{6}$ There is a substantial risk of nephrotoxicity with use of colistin that

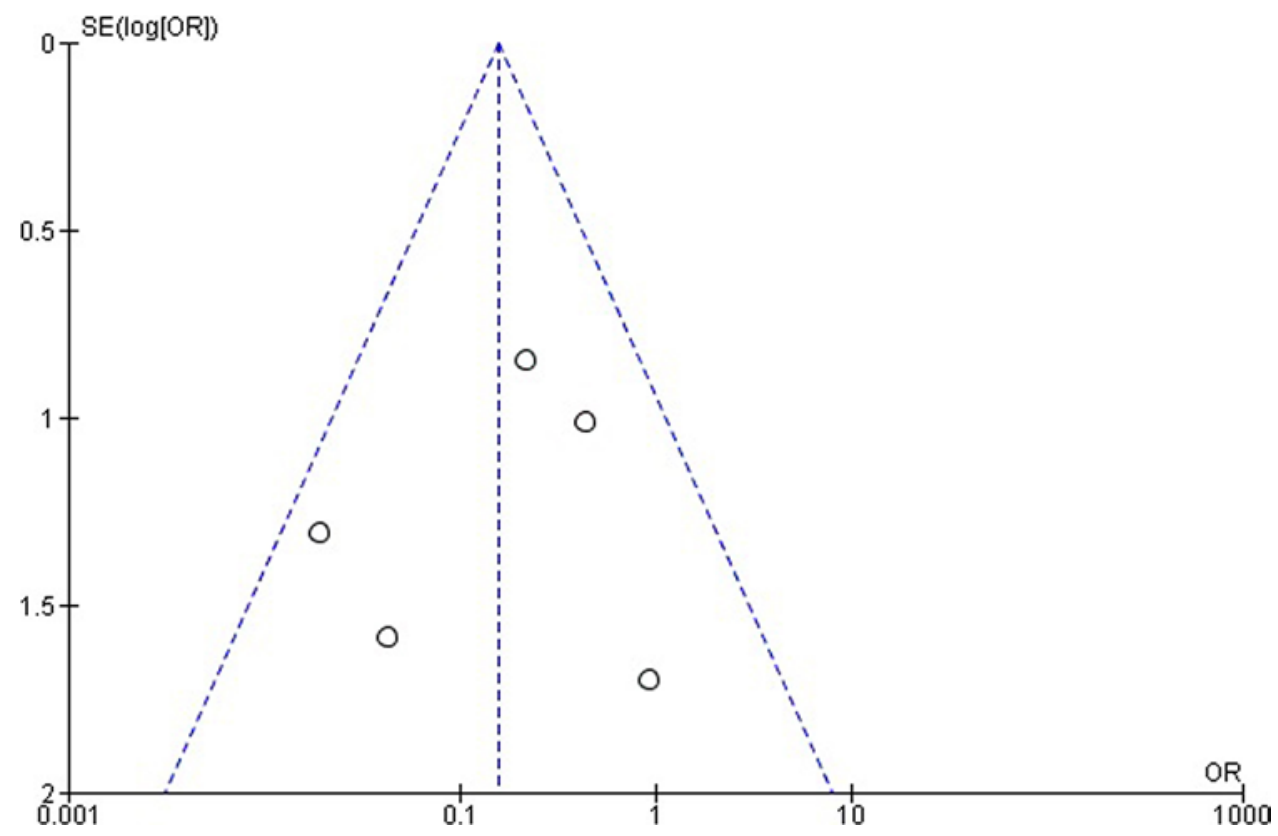

FIG. 2. Funnel plot showing the distribution of studies for assessment of bias. SE = standard error. 
TABLE 2. Mortality rates in different subgroups of patients with MDR A. baumannii

\begin{tabular}{|c|c|c|c|c|c|}
\hline Group & Fotakopoulos et al., 2016 & De Bonis et al., 2016 & Tuon et al., 2010 & Rodriguez et al., 2008 & Moon et al., 2013 \\
\hline \multicolumn{6}{|l|}{ IV group } \\
\hline No. of patients & 9 & 9 & 15 & 29 & 11 \\
\hline No. of deaths & 8 & 5 & 13 & 11 & 4 \\
\hline \multicolumn{6}{|l|}{ IV + IT group } \\
\hline No. of patients & 15 & 9 & 2 & 17 & 10 \\
\hline No. of deaths & 2 & 0 & 2 & 2 & 2 \\
\hline Time to CSF sterilization in days & NA & $21(8-48)$ & NA & 21 & NA \\
\hline Overall mortality, no. (\%) & $10(41.6)$ & $5(27.7)$ & $15(78)$ & $13(28.2)$ & $6(28.5)$ \\
\hline
\end{tabular}

may require dosage reduction and further jeopardize the outcome of treatment. ${ }^{5}$ This drug has also been linked to neurotoxicity. Polymyxins can cause neuromuscular blockade due to inhibition of acetylcholine release. The majority of postneurosurgical A. baumannii infections are associated with indwelling ventriculostomy tubes or CSF leaks. ${ }^{24}$ The median time for development of postoperative meningitis is 12 days (range 1-40 days). The risk factors predisposing patients to postneurosurgical A. baumannii meningitis are CSF leaks, ${ }^{11}$ prolonged external ventricular drainage, ${ }^{12}$ prolonged duration of surgery, ${ }^{11}$ opening of sinus during surgery, ${ }^{12}$ wound infection, ${ }^{11}$ repeat surgery, ${ }^{22}$ and critical illness. ${ }^{12}$

The 2017 Infectious Diseases Society of America's guidelines ${ }^{25}$ state that intraventricular antimicrobial therapy should be considered in patients with health care-associated meningitis who respond poorly to systemic therapy. It is also recommended to clamp the ventricular or intrathecal drain for a period of 15-60 minutes to allow the drug to achieve an equilibrium. The actual intraventricular dosage is determined by calculating the "inhibitory quotient." After administering the first dose, the trough levels of antibiotic are determined from the sample of CSF taken just before giving the second dose. The inhibitory quotient is calculated by dividing the trough level by the minimum inhibitory concentration for the organism. The inhibitory quotient should exceed 10-20 for consistent CSF sterilization. The dosage also needs adjustment depending on the size of the ventricle and daily output from the drain. For the infected CSF drain or shunt tube, removal is recommended.

The recommended doses of colistin for intraventricular administration are $5 \mathrm{mg}$ or $62,500 \mathrm{IU}$ per day $(1 \mathrm{mg}$ of $\mathrm{CMS}=12,500 \mathrm{IU}$ ) in adults and $2 \mathrm{mg}$ or $25,000 \mathrm{IU}$ per day in children. ${ }^{25}$ The recommended pediatric dosage of CMS per day is between 50,000 and 75,000 IU/kg/day (4-6 mg/kg of body weight/day) in Europe and 83,000$166,000 \mathrm{IU} / \mathrm{kg} /$ day $\left(6.6-13.3 \mathrm{mg} / \mathrm{kg} /\right.$ day) in the US. ${ }^{13}$ The British Society for Antimicrobial Chemotherapy Working Party recommended that the initial intraventricular dose should be based on the ventricular volume as determined by imaging. ${ }^{3}$ To avoid increasing intracranial pressure before drug instillation, an equal volume of CSF must be removed. After instillation of the drug, a saline flush is done to minimize the amount of drug left over in the drain tube. The patient's neurological status must be monitored both before and after giving the drug via the intraventricular route.

De Bonis et al. ${ }^{4}$ reported a CSF sterilization rate of $33 \%$ in the IV-only group and $100 \%$ in the IV + IT group $(\mathrm{p}=$ 0.009). The mean time for CSF sterilization was 21 days. Tuon et al. ${ }^{26}$ reported a high mortality rate of $72.7 \%$. Only 2 patients in their study received intrathecal therapy. Two patients who received IV + IT therapy also died. It is interesting to note that only $69 \%$ of patients who died received appropriate, sensitive antibiotics. Thus, an inappropriate antibiotic regimen could have led to the ineffectiveness of IV + IT therapy in this study. Transmission of infec-

\begin{tabular}{|c|c|c|c|c|c|c|c|c|c|c|}
\hline \multirow[b]{2}{*}{ Study or Subgroup } & \multicolumn{2}{|c|}{ IV+IT GROUP } & \multicolumn{2}{|c|}{ IV ONLY GROUP } & \multirow[b]{2}{*}{ Weight } & \multirow{2}{*}{$\begin{array}{c}\text { Odds Ratio } \\
\text { M-H, Fixed, 95\% Cl }\end{array}$} & \multirow{2}{*}{\multicolumn{4}{|c|}{$\begin{array}{c}\text { Odds Ratio } \\
\text { M-H, Fixed, } 95 \% \mathrm{Cl}\end{array}$}} \\
\hline & Events & Total & Events & Total & & & & & & \\
\hline Fotakopoulos 2016 & 2 & 15 & 8 & 9 & $34.9 \%$ & $0.02[0.00,0.25]$ & & & & \\
\hline De Bonis 2016 & 0 & 9 & 5 & 9 & $21.0 \%$ & $0.04[0.00,0.96]$ & & & & \\
\hline Rodriguez 2008 & 2 & 17 & 11 & 29 & $28.9 \%$ & $0.22[0.04,1.14]$ & & & & \\
\hline Moon 2013 & 2 & 10 & 4 & 11 & $12.3 \%$ & $0.44[0.06,3.16]$ & & & & \\
\hline Tuon 2010 & 2 & 2 & 13 & 15 & $2.9 \%$ & $0.93[0.03,25.68]$ & & & & \\
\hline Total $(95 \% \mathrm{Cl})$ & & 53 & & 73 & $100.0 \%$ & $0.16[0.06,0.40]$ & & & & \\
\hline Total events & 8 & & 41 & & & & & & & \\
\hline $\begin{array}{l}\text { Heterogeneity: } \mathrm{Chi}^{2}= \\
\text { Test for overall effect }\end{array}$ & $\begin{array}{l}5.53, \mathrm{df}= \\
\mathrm{Z}=3.90(\end{array}$ & $\begin{array}{l}+P=0 \\
<0.00\end{array}$ & $\begin{array}{l}\text { 24); }\left.\right|^{2}=2 \\
\text { 01) }\end{array}$ & & & & $\stackrel{\longmapsto}{\circ} .001$ & 0.1 & $1 \begin{array}{r}10 \\
\text { IV ONLY }\end{array}$ & 1000 \\
\hline
\end{tabular}

FIG. 3. Forest plot with mortality as the outcome showing the calculated odds ratios, weights, and confidence interval of each study, and the overall pooled odds ratio. The event here is the number of deaths that have occurred in the respective groups. $\mathrm{M}-\mathrm{H}=$ Mantel-Haenszel. 
tion from other sources like the lungs and urinary tract is a concern in the ICU setting. Fotakopoulos et al. ${ }^{7}$ found that $5.6 \%$ of external ventricular drains were infected with A. baumannii.

The importance of strict adherence to hand hygiene and hospital infection protocols cannot be overemphasized. It must be remembered that starting IV + IT therapy does not guarantee the survival of patients. In the studies reviewed, 8 deaths occurred in IV + IT group. What has caused the deaths in these cases? This could be explained in part due to late initiation of intraventricular therapy. It must be inferred that in usual clinical practice, IV therapy is started first, and when there is no improvement, then as a last resort IV + IT therapy is started. We challenge this notion and hypothesize that this may lead to worse outcomes. The other reason could be the variation in the sensitivity patterns of the organism to the antibiotics. Moon et al..$^{18}$ found that carbapenem-resistant strains had a higher mortality than other strains. It is not possible to answer this question based on the current study; randomized controlled trials are needed to address these issues. In the absence of clear guidelines, clinicians are faced with a difficult dilemma in caring for these patients. In the situation of clinical nonimprovement with systemic therapy, it is prudent to get intrathecal or intraventricular access and commence the therapy at the earliest possible time. If sensitivity is demonstrated only for colistin, then we recommend that IV + IT therapy be started at the earliest opportunity.

\section{Limitations of the Study}

Our study has limitations in evaluating a small number of observational studies, the results of all of which are in favor of IV + IT therapy. However, all the studies have limited sample size, with considerable variability in the mortality rates. Hence, we felt a need to assess the overall impact of IV + IT therapy on the mortality rate in a larger cohort, and to establish its unequivocal role in reducing mortality. Further questions like optimum intraventricular dosage, duration of the treatment, and the treatment strategy in pan-drug-resistant infections need serious attention.

\section{Conclusions}

The data analyzed from this study show that the inclusion of intrathecal or intraventricular antimicrobial therapy with intravenous therapy is associated with an $84 \%$ lower chance of mortality in postneurosurgical infections due to A. baumannii. The intrathecal route should be strongly considered when dealing with postneurosurgical A. baumannii infections.

\section{References}

1. Alsan M, Klompas M: Acinetobacter baumannii: an emerging and important pathogen. J Clin Outcomes Manag 17:363-369, 2010

2. Antunes LC, Visca P, Towner KJ: Acinetobacter baumannii: evolution of a global pathogen. Pathog Dis 71:292-301, 2014

3. Brown EM, de Louvois J, Lees PD, Pople IK: The management of neurosurgical patients with postoperative bacterial or aseptic meningitis or external ventricular drainassociated ventriculitis. Br J Neurosurg 14:7-12, 2000
4. De Bonis P, Lofrese G, Scoppettuolo G, Spanu T, Cultrera R, Labonia M, et al: Intraventricular versus intravenous colistin for the treatment of extensively drug resistant Acinetobacter baumannii meningitis. Eur J Neurol 23:68-75, 2016

5. Duncan DA: Colistin toxicity. Neuromuscular and renal manifestations. Two cases treated by hemodialysis. Minn Med 56:31-35, 1973

6. Falagas ME, Kasiakou SK: Toxicity of polymyxins: a systematic review of the evidence from old and recent studies. Crit Care 10:R27, 2006

7. Fotakopoulos G, Makris D, Chatzi M, Tsimitrea E, Zakynthinos E, Fountas K: Outcomes in meningitis/ ventriculitis treated with intravenous or intraventricular plus intravenous colistin. Acta Neurochir (Wien) 158:603-610, 2016

8. James HE, Wilson HD, Connor JD, Walsh JW: Intraventricular cerebrospinal fluid antibiotic concentrations in patients with intraventricular infections. Neurosurgery 10:50-54, 1982

9. Jiménez-Mejías ME, Becerril B, Márquez-Rivas FJ, Pichardo C, Cuberos L, Pachón J: Successful treatment of multidrug-resistant Acinetobacter baumannii meningitis with intravenous colistin sulfomethate sodium. Eur J Clin Microbiol Infect Dis 19:970-971, 2000

10. Karaiskos I, Galani L, Baziaka F, Giamarellou H: Intraventricular and intrathecal colistin as the last therapeutic resort for the treatment of multidrug-resistant and extensively drug-resistant Acinetobacter baumannii ventriculitis and meningitis: a literature review. Int J Antiomicrob Agents 41:499-508

11. Korinek AM, Baugnon T, Golmard JL, van Effenterre R, Coriat P, Puybasset L: Risk factors for adult nosocomial meningitis after craniotomy: role of antibiotic prophylaxis. Neurosurgery 59:126-133, 2006

12. Kourbeti IS, Jacobs AV, Koslow M, Karabetsos D, Holzman RS: Risk factors associated with postcraniotomy meningitis. Neurosurgery 60:317-326, 2007

13. Li J, Nation RL, Turnidge JD, Milne RW, Coulthard K, Rayner CR, et al: Colistin: the re-emerging antibiotic for multidrug-resistant Gram-negative bacterial infections. Lancet Infect Dis 6:589-601, 2006

14. Lin MF, Lan CY: Antimicrobial resistance in Acinetobacter baumannii: from bench to bedside. World J Clin Cases 2:787-814, 2014

15. Magiorakos AP, Srinivasan A, Carey RB, Carmeli Y, Falagas $\mathrm{ME}$, Giske CG, et al: Multidrug-resistant, extensively drugresistant and pandrug-resistant bacteria: an international expert proposal for interim standard definitions for acquired resistance. Clin Microbiol Infect 18:268-281, 2012

16. Markantonis SL, Markou N, Fousteri M, Sakellaridis N, Karatzas S, Alamanos I, et al: Penetration of colistin into cerebrospinal fluid. Antimicrob Agents Chemother 53:4907-4910, 2009

17. Metan G, Alp E, Aygen B, Sumerkan B: Acinetobacter baumannii meningitis in post-neurosurgical patients: clinical outcome and impact of carbapenem resistance. J Antimicrob Chemother 60:197-199, 2007

18. Moon C, Kwak YG, Kim BN, Kim ES, Lee CS: Implications of postneurosurgical meningitis caused by carbapenemresistant Acinetobacter baumannii. J Infect Chemother 19:916-919, 2013

19. Nau R, Sörgel F, Eiffert H: Penetration of drugs through the blood-cerebrospinal fluid/blood-brain barrier for treatment of central nervous system infections. Clin Microbiol Rev 23:858-883, 2010

20. Peleg AY, Seifert H, Paterson DL: Acinetobacter baumannii: emergence of a successful pathogen. Clin Microbiol Rev 21:538-582, 2008

21. Perez F, Hujer AM, Hujer KM, Decker BK, Rather PN, 
Bonomo RA: Global challenge of multidrug-resistant Acinetobacter baumannii. Antimicrob Agents Chemother 51:3471-3484, 2007

22. Reichert MC, Medeiros EA, Ferraz FA: Hospital-acquired meningitis in patients undergoing craniotomy: incidence, evolution, and risk factors. Am J Infect Control 30:158-164, 2002

23. Rodriguez Guardado A, Blanco A, Asensi V, Perez F, Rial JC, Pintado V, et al: Multidrug-resistant Acinetobacter meningitis in neurosurgical patients with intraventricular catheters: assessment of different treatments. J Antimicrob Chemother 61:908-913, 2008

24. Siegman-Igra Y, Bar-Yosef S, Gorea A, Avram J: Nosocomial acinetobacter meningitis secondary to invasive procedures: report of 25 cases and review. Clin Infect Dis 17:843-849, 1993

25. Tunkel AR, Hasbun R, Bhimraj A, Byers K, Kaplan SL, Michael Scheld W, et al: 2017 Infectious Diseases Society of America's clinical practice guidelines for healthcareassociated ventriculitis and meningitis. Clin Infect Dis 64:e34-e65, 2017

26. Tuon FF, Penteado-Filho SR, Amarante D, Andrade MA, Borba LA: Mortality rate in patients with nosocomial Acinetobacter meningitis from a Brazilian hospital. Braz J Infect Dis 14:437-440, 2010
27. Villegas MV, Hartstein AI: Acinetobacter outbreaks, 19772000. Infect Control Hosp Epidemiol 24:284-295, 2003

\section{Disclosures}

The authors report no conflict of interest concerning the materials or methods used in this study or the findings specified in this paper.

\section{Author Contributions}

Conception and design: Nanda, Mohammed, Savardekar. Acquisition of data: Mohammed, Narayan. Analysis and interpretation of data: Mohammed. Drafting the article: Mohammed, Savardekar, Patra, Narayan. Critically revising the article: all authors. Reviewed submitted version of manuscript: all authors. Approved the final version of the manuscript on behalf of all authors: Nanda. Statistical analysis: Mohammed, Narayan. Administrative/technical/material support: Nanda, Savardekar, Patra. Study supervision: Nanda.

\section{Correspondence}

Anil Nanda, Department of Neurosurgery, Louisiana State University Health Sciences Center, 1501 Kings Highway, Shreveport, LA 71130.email: ananda@1suhsc.edu. 